\title{
Depth of thermal penetration in straight grinding
}

\author{
J. L. González-Santander • H. \\ Espinós-Morató
}

Received: date / Accepted: date

\begin{abstract}
Unlike the usual numerical FEM approach to determine the thermally affected layer during the grinding process, we propose a simple analytical approach to estimate the depth of thermal penetration. For this purpose, the one-dimensional definition of depth of thermal penetration is applied to the two-dimensional heat transfer models of straight grinding. A method for computing the depth of thermal penetration in these two-dimensional models is derived and compared to the one-dimensional approximation. For dry grinding, it turns out that the one-dimensional approximation is quite accurate when we consider a moderate percentage in the temperature fall beneath the surface, regardless the type of heat flux profile entering into the workpiece (i.e. constant, linear, triangular or parabolic). In wet grinding, the latter is true if we consider a constant heat flux profile and a high Peclet number, i.e. Pe $>5$. Finally, the one- and two-dimensional approaches calculating analytically the depth of thermal penetration have been compared to the temperature field numerically evaluated by a three-dimensional FEM simulation given in the literature, obtaining a quite good agreement.
\end{abstract}

Keywords depth of thermal penetration · heat transfer in grinding

\section{Introduction}

Straight grinding is an industrial machining process to produce a smooth finish on the flat surface of a workpiece. In this process, there are abrasive grains adhered to the peripheral area of the grinding wheel, thereby when this one

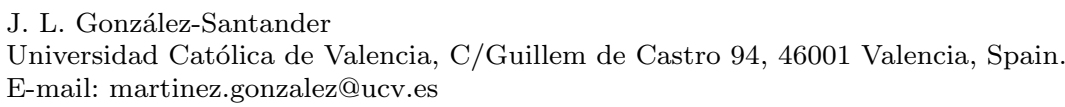


rotates at high speed, it removes the surface layer of the workpiece. In Fig. 1, we consider the grinding wheel fixed with respect to the observer and the workpiece moving from right to left at feed rate $v_{d}$. For not very conductive workpieces, almost all the energy of the process is converted into heat, which is concentrated within the grinding area of width $2 \ell$ (see Ref. [1, Chap.6]). This heat flux entering into the workpiece leads to high temperatures that can damage the workpiece (i.e. burning, phase transformations or residual stresses), thus many times coolant is applied onto the surface to avoid thermally-induced damage.

From analytical methods, Ref. [2], FEM analysis, Ref. [3], and experimental measurements, Ref. [4], of the temperature field in grinding, it is known that the temperature field is very shallow, thereby it exists a high gradient of temperature nearby the surface. In one-dimensional heat transfer, there exist some definitions of the depth of thermal penetration, according to different boundary conditions, Ref. [5]. We will analyze these definitions in Section 4.2. Anyway, in the literature, there seems to be a lack of an analytic method for evaluating the characteristic depth of the temperature field in grinding from the thermal properties of the workpiece and the parameters of the process. For instance, when burnout occurs, an analytical approximation of the depth of thermal penetration would be useful to predict the thickness of the surface layer that has suffered a change of phase, as well as the thickness of the thermal induced hardness distribution beneath the ground surface, Ref. [6]. This hardness distribution has been studied thoroughly in the literature regarding the grind-hardening process, Ref. [7]. The usual approach to study the heat treated zone in grind-hardening is based in FEM analysis, Ref. [8]. However, in this article, we propose a very simple analytical approach that turns out to be applicable in many cases and that is extremely easy to compute. In particular, we will evaluate when and how the one-dimensional approach of the depth of thermal penetration can be applied to straight surface grinding. For this purpose, Section 2 is devoted to the equations and boundary conditions governing the heat transfer in grinding. Then, Section 3 analyzes how these two-dimensional boundary-value problems can be approximated by one-dimensional problems in order to estimate the depth of thermal penetration, both in dry as in wet grinding. Next, in Section 4, the depth of thermal penetration is calculated from the analytical results for the temperature field in the stationary regime, considering the most common heat flux profiles reported in the literature, i.e. constant, linear, triangular and parabolic. Section 5 presents some numerical examples for different grinding regimes and workpiece materials, comparing the two-dimensional depth of thermal penetration to the one-dimensional approximation. Also, we will compare one- and two-dimensional approaches of the depth of thermal penetration to a temperature field found in the literature computed by using a three-dimensional FEM analysis in dry grinding. Finally, the conclusions are summarized in Section 6. 


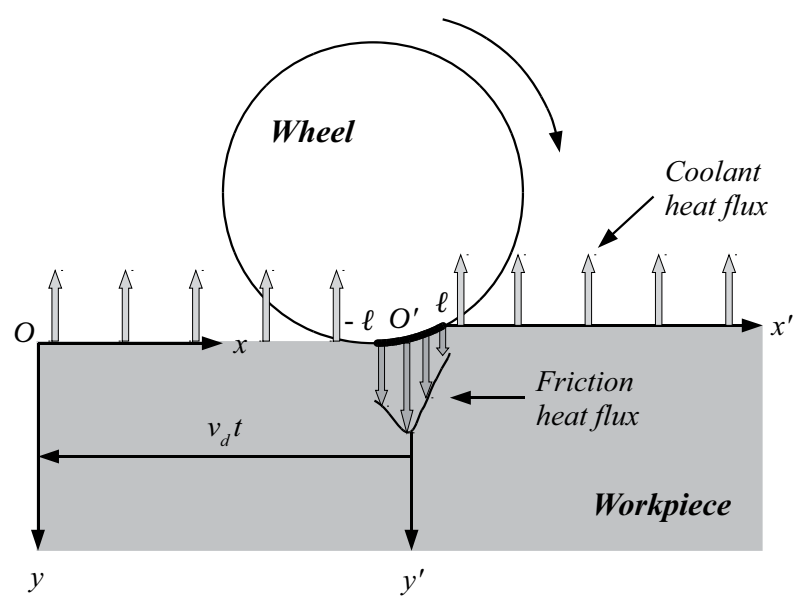

Fig. 1 Grinding setup.

\begin{tabular}{|c|c|c|}
\hline Symbol & Meaning & SI units \\
\hline \hline$k_{0}$ & Thermal conductivity & $\mathrm{W} \mathrm{m}^{-1} \mathrm{~K}^{-1}$ \\
\hline$k$ & Thermal diffusivity & $\mathrm{m}^{2} \mathrm{~s}^{-1}$ \\
\hline$T$ & Workpiece temperature & $\mathrm{K}$ \\
\hline$T_{\infty}$ & Room temperature & $\mathrm{K}$ \\
\hline$v_{d}$ & Feedrate & $\mathrm{m} \mathrm{s}^{-1}$ \\
\hline $2 \ell$ & Contact length & $\mathrm{m}$ \\
\hline$q$ & Average heat flux within contact length & $\mathrm{W} \mathrm{m}^{-2}$ \\
\hline$h$ & Heat transfer coefficient & $\mathrm{W} \mathrm{m}^{-2} \mathrm{~K}^{-1}$ \\
\hline
\end{tabular}

Table 1 Nomenclature of symbols.

\section{The equations}

Usually, the heat transfer in surface grinding is modeled in a two-dimensional frame in which friction due to the grinding wheel is represented by a moving heat band source sliding over the workpiece surface (see Ref. [2]). Since the depth of cut is very small with respect to the contact length $2 \ell$ in surface grinding, the heat source is assumed to be flat (see Fig. 1). Also in Fig. 1, two Cartesian coordinate systems are shown. The $X Y$ coordinate system is fixed to the workpiece, and the $X^{\prime} Y^{\prime}$ coordinate system is fixed to the wheel, being the origin $O^{\prime}$ located in the center of the contact length. The relationship between both systems is

$$
\begin{aligned}
& x^{\prime}=x-v_{d} t, \\
& y^{\prime}=y,
\end{aligned}
$$

thereby, at $t=0$, they overlap each other. The temperature field of the workpiece in the $X^{\prime} Y^{\prime}$ coordinate system is denoted by $T\left(t, x^{\prime}, y^{\prime}\right)$, and must satisfy the following convective heat equation, Ref. [9, Eq. 1.7(2)], (see Table 1 for 
the nomenclature used),

$$
\begin{aligned}
& \frac{\partial T\left(t, x^{\prime}, y^{\prime}\right)}{\partial t} \\
= & k\left(\frac{\partial^{2} T\left(t, x^{\prime}, y^{\prime}\right)}{\partial x^{\prime 2}}+\frac{\partial^{2} T\left(t, x^{\prime}, y^{\prime}\right)}{\partial y^{\prime 2}}\right)+v_{d} \frac{\partial T\left(t, x^{\prime}, y^{\prime}\right)}{\partial x^{\prime}}
\end{aligned}
$$

Notice that the heat conduction occurs in the half-plane $-\infty<x^{\prime}<$ $\infty, y^{\prime} \geq 0$ for $t \geq 0$. It is worth noting that in the stationary regime, the temperature field $T\left(x^{\prime}, y^{\prime}\right)$ does not evolve in time, i.e. $\partial T / \partial t=0$, thus Eq. (2) is reduced to

$$
k\left(\frac{\partial^{2} T\left(x^{\prime}, y^{\prime}\right)}{\partial x^{\prime 2}}+\frac{\partial^{2} T\left(x^{\prime}, y^{\prime}\right)}{\partial y^{\prime 2}}\right)+v_{d} \frac{\partial T\left(x^{\prime}, y^{\prime}\right)}{\partial x^{\prime}}=0
$$

Regarding the initial condition, we assume that before the grinding process starts, the workpiece is at room temperature $T_{\infty}$, hence

$$
T\left(0, x^{\prime}, y^{\prime}\right)=T_{\infty}
$$

Also, the boundary condition provides the heat flux balance on the surface of the workpiece (i.e. $y^{\prime}=0$ ), and according to Ref. [10] is given by

$$
k_{0} \frac{\partial T\left(t, x^{\prime}, 0\right)}{\partial y^{\prime}}=b\left(t, x^{\prime}\right)\left[T\left(t, x^{\prime}, 0\right)-T_{\infty}\right]+d\left(t, x^{\prime}\right),
$$

The first term of Eq. (5) models the application of lubricant over the surface of the workpiece, denoting $b\left(t, x^{\prime}\right)$ as the heat transfer coefficient. In this article we will consider only the cases of dry grinding, i.e. $b\left(t, x^{\prime}\right)=0$, and wet grinding with a constant heat transfer coefficient, i.e. $b\left(t, x^{\prime}\right)=h$. Although the actual application of coolant is not uniform over the workpiece surface, the selection of a constant heat transfer coefficient makes the boundary-value problem given in Eqs. (2), (4) tractable, and analytical solutions exist in the literature. Moreover, it is known that the coolant convection is negligible outside the grinding zone (see Ref. [1, p. 168]). In view of the first term of Eq. (5), the latter is clear since the heat removed by the coolant is greater where the surface temperature is higher. Therefore, the adequate value of $h$ to consider in the model is the one that actually acts in the grinding zone. Due to the rotation of the wheel, the action of the coolant is supposed to be uniform along the contact length, thus a constant $h$ assumption within the grinding zone seems to be reasonably realistic.

The second term of Eq. (5) contains the $d\left(t, x^{\prime}\right)$ function, which represents the heat flux entering into the workpiece due to friction in the contact zone between the wheel and the workpiece. In this article we will consider only continuous grinding, for which friction does not vary over time, hence

$$
d\left(t, x^{\prime}\right)=-q f\left(x^{\prime}\right) \theta\left(x^{\prime}+\ell\right) \theta\left(\ell-x^{\prime}\right),
$$


where $\theta(x)$ denotes the Heaviside function, and $f(x)$ is a dimensionless function that takes into account the type of heat flux profile we have within the contact zone. Also, $f(x)$ is normalized to unity as follows,

$$
\frac{1}{2 \ell} \int_{-\ell}^{\ell} f\left(x^{\prime}\right) d x^{\prime}=1 .
$$

Table 2 shows the most common heat flux profiles reported in the literature: constant, Refs. [11,12]; linear, Refs. [13,14]; triangular, Refs. [15,4]; and parabolic, Ref. [16]. Note that in the triangular case, $\lambda$ is a dimensionless quantity that locates the apex of the heat flux profile (see Fig. 2).

\begin{tabular}{|c|c|}
\hline Heat flux profile & $f\left(x^{\prime}\right)$ \\
\hline \hline Constant & 1 \\
Linear & $1+\frac{x^{\prime}}{\ell}$ \\
Triangular & $2\left(1+\frac{x^{\prime} / \ell-\lambda}{\lambda+\operatorname{sign}\left(\lambda-x^{\prime} / l\right)}\right)$ \\
Parabolic & $\frac{3}{4}\left(1+\frac{x^{\prime}}{\ell}\right)^{2}$ \\
\hline
\end{tabular}

Table 2 Heat flux profiles for continuous grinding.

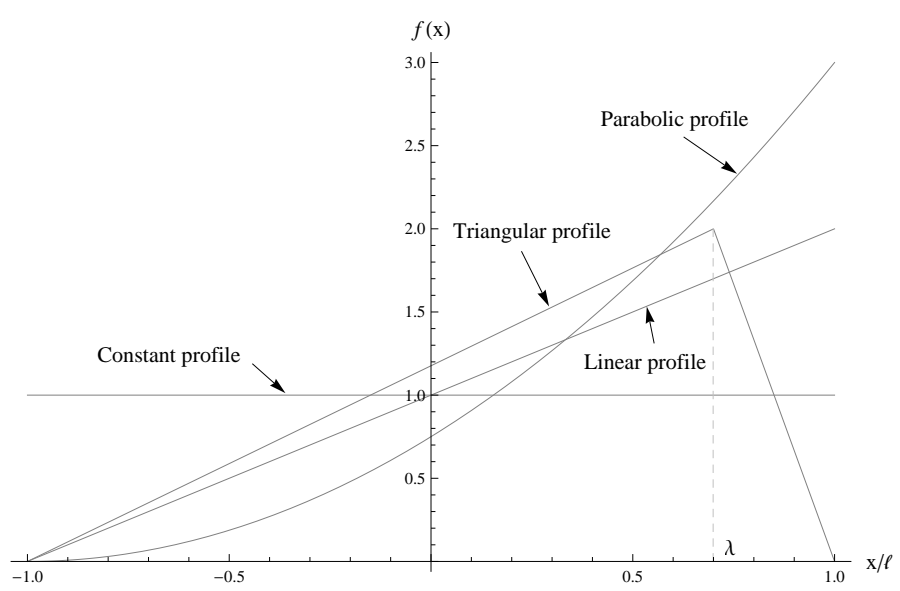

Fig. 2 Heat flux profiles considered.

Note that performing the change of variables in Eq. (1), the convective term in Eq. (2) disappears in the $X^{\prime} Y^{\prime}$ coordinate system, since there is no 
convection when we are moving with the workpiece. Therefore,

$$
\frac{\partial T(t, x, y)}{\partial t}=k\left(\frac{\partial^{2} T(t, x, y)}{\partial x^{2}}+\frac{\partial^{2} T(t, x, y)}{\partial y^{2}}\right) .
$$

Also, the initial condition given in Eq. (4) remains equal,

$$
T(0, x, y)=T_{\infty} .
$$

\section{One-dimensional approach}

Let us consider the boundary condition given in Eq. (5) for dry grinding, so that the heat transfer coefficient over the surface is null, i.e. $b\left(t, x^{\prime}\right)=0$. Moreover, consider that the heat flux entering into the workpiece is constant over the grinding area, i.e. $f\left(x^{\prime}\right)=1$ in Eq. (6), thus

$$
k_{0} \frac{\partial T}{\partial y}\left(t, x^{\prime}, 0\right)=-q \theta\left(x^{\prime}+\ell\right) \theta\left(\ell-x^{\prime}\right),
$$

If we refer Eq. (10) to the $X Y$ coordinate system, we will have

$$
k_{0} \frac{\partial T}{\partial y}(t, x, 0)=-q \theta\left(x-v_{d} t+\ell\right) \theta\left(\ell-x+v_{d} t\right),
$$

that is to say, the heat source is now moving over the surface at velocity $v_{d}$. Therefore, roughly speaking, the heat source heats up a given fixed point $(x, y)$ of the workpiece during the following characteristic time,

$$
t_{c}=\frac{2 \ell}{v_{d}} .
$$

For high Peclet numbers, namely Pe $>5$ (see Table 3 for the definition of Peclet number), we can neglect the heat transfer along the $x$-axis (see Ref. [17]), thus Eq. (8) can be approximated by the one-dimensional heat equation,

$$
\frac{\partial T(t, y)}{\partial t} \approx k \frac{\partial^{2} T(t, y)}{\partial y^{2}} .
$$

Also, during time $t_{c}$, the boundary condition given in Eq. (11) can be approximated by

$$
k_{0} \frac{\partial T}{\partial y}(t, 0) \approx-q .
$$

For wet grinding, consider in the boundary condition given in Eq. (5) a constant heat transfer coefficient over the surface, i.e. $b\left(t, x^{\prime}\right)=h$. Performing the same reasoning as above, we have the same one-dimensional heat equation as in Eq. (13), and during time $t_{c}$, the boundary condition can be approximated by

$$
k_{0} \frac{\partial T}{\partial y}(t, 0) \approx-q+h\left[T(t, 0)-T_{\infty}\right] .
$$


According to Ref. [5], the depth of thermal penetration $\delta_{p}(t)$ for onedimensional heat equation is defined as the depth at which the temperature rise falls to $1 \%$ with respect to the temperature rise at the boundary. This definition can be generalized easily to a decay to $p \%$ of the temperature rise as follows,

$$
\frac{T\left(t, \delta_{p}(t)\right)-T_{\infty}}{T(t, 0)-T_{\infty}}=p .
$$

According to Eq. (16), the depth of thermal penetration depends on $t, p$ and the kind of boundary condition, namely Eqs. (14) or (15). Since these boundary conditions have been approximated from the two-dimensional approach considering that the heat source is acting on the workpiece surface during time $t_{c}$, hereafter we will apply Eq. (16) defining the depth of thermal penetration $\delta_{p}$ at time $t_{c}=2 \ell / v_{d}$,

$$
\delta_{p}=\delta_{p}\left(t_{c}\right)
$$

\subsection{Dry grinding}

As aforementioned in Eqs. (9), (13) and (14), we have to solve the onedimensional heat equation,

$$
\frac{\partial T_{\mathrm{dry}}(t, y)}{\partial t}=k \frac{\partial^{2} T_{\mathrm{dry}}(t, y)}{\partial y^{2}},
$$

subjected to a boundary condition of the second kind, Ref. [18, Sect.1.12],

$$
k_{0} \frac{\partial T_{\text {dry }}}{\partial y}(t, 0)=-q, \quad t>0
$$

and to the following initial condition given in a semi-infinite region,

$$
T_{\text {dry }}(0, y)=T_{\infty}, \quad y>0
$$

The solution of the above problem is given in Ref. [9, Eqn. 2.9(7)], and it reads as,

$$
\begin{aligned}
& T_{\text {dry }}(t, y) \\
= & T_{\infty}+\frac{2 q}{k_{0}}\left[\sqrt{\frac{k t}{\pi}} \exp \left(-\frac{y^{2}}{4 k t}\right)-\frac{y}{2} \operatorname{erfc}\left(\frac{y}{2 \sqrt{k t}}\right)\right],
\end{aligned}
$$

thus the temperature on the surface is

$$
T_{\text {dry }}(t, 0)=T_{\infty}+\frac{2 q}{k_{0}} \sqrt{\frac{k t}{\pi}}
$$


Thereby, according to the depth of thermal penetration definition given in Eqs. (16) and (17), and the results given in Eqs. (21) and (22), we have

$$
\begin{aligned}
& \frac{T_{\mathrm{dry}}\left(t_{c}, \delta_{p}\right)-T_{\infty}}{T_{\mathrm{dry}}\left(t_{c}, 0\right)-T_{\infty}} \\
= & \exp \left(-\frac{\delta_{p}^{2}}{4 k t_{c}}\right)-\frac{\sqrt{\pi} \delta_{p}}{2 \sqrt{k t_{c}}} \operatorname{erfc}\left(\frac{\delta_{p}}{2 \sqrt{k t_{c}}}\right)=p .
\end{aligned}
$$

In order to solve for $\delta_{p}$, consider the following dimensionless quantity

$$
\Delta=\frac{\delta_{p}}{2 \sqrt{k t_{c}}},
$$

so that Eq. (23) is rewritten as

$$
g(\Delta)=e^{-\Delta^{2}}-\sqrt{\pi} \Delta \operatorname{erfc}(\Delta)=p .
$$

Hence, taking into account Eq. (12), the depth of thermal penetration is given by

$$
\delta_{p}=2 \sqrt{k t_{c}} g^{-1}(p),
$$

where the inverse function $g^{-1}$ can be computed numerically very easily with a root searching method like Newton-Raphson.

\subsection{Wet grinding}

As mentioned before in Eqs. (9), (13) and (15), we have to solve the onedimensional heat equation,

$$
\frac{\partial T_{\mathrm{wet}}(t, y)}{\partial t}=k \frac{\partial^{2} T_{\mathrm{wet}}(t, y)}{\partial y^{2}},
$$

subjected to the following initial condition in a semi-infinite region,

$$
T_{\text {wet }}(0, y)=T_{\infty}, \quad y>0,
$$

and to a combined boundary condition of the second and third kinds, Ref. [18, Sect.1.12],

$$
\frac{\partial T_{\mathrm{wet}}}{\partial x}(t, 0)=-q_{0}+h_{0}\left[T_{\mathrm{wet}}(t, 0)-T_{\infty}\right], \quad t>0,
$$

where we have set

$$
\begin{aligned}
q_{0} & =\frac{q}{k_{0}}, \\
h_{0} & =\frac{h}{k_{0}} .
\end{aligned}
$$


The solution to the problem stated in Eqs. (27)-(29) does not seem to be reported in the most common literature. However, the solution can be calculated as (see the Appendix for details)

$$
\begin{aligned}
& T_{\text {wet }}(t, y) \\
= & T_{\infty}+\frac{q_{0}}{h_{0}} \\
& \times\left\{\operatorname{erfc}\left(\frac{y}{2 \sqrt{k t}}\right)-e^{h_{0}^{2} k t+h_{0} y} \operatorname{erfc}\left(\frac{y}{2 \sqrt{k t}}+h_{0} \sqrt{k t}\right)\right\},
\end{aligned}
$$

so that the temperature on the surface is

$$
T_{\text {wet }}(t, 0)=T_{\infty}+\frac{q_{0}}{h_{0}}\left\{1-e^{h_{0}^{2} k t} \operatorname{erfc}\left(h_{0} \sqrt{k t}\right)\right\}
$$

As a consistency test, it is worth noting that performing the limit $h_{0} \rightarrow 0$ in Eq. (32) (i.e. no coolant is applied onto the surface), we recover the solution given in Eq. (21) for dry grinding. Indeed, according to Taylor's first order expansion of the erfc $\left(z_{0}+z\right)$ and $e^{z}$ functions,

$$
\begin{aligned}
\operatorname{erfc}\left(z_{0}+z\right) & \approx \operatorname{erfc}\left(z_{0}\right)-\frac{2 z}{\sqrt{\pi}} e^{-z_{0}^{2}}, \quad z \rightarrow 0 \\
e^{z} & \approx 1+z, \quad z \rightarrow 0
\end{aligned}
$$

we have,

$$
\begin{aligned}
& \lim _{h_{0} \rightarrow 0} T_{\text {wet }}(t, y)-T_{\infty} \\
=\lim _{h_{0} \rightarrow 0} & \frac{q_{0}}{h_{0}}\left\{\operatorname{erfc}\left(\frac{y}{2 \sqrt{k t}}\right)-\left(1+h_{0} y\right)\right. \\
& \left.\times\left[\operatorname{erfc}\left(\frac{y}{2 \sqrt{k t}}\right)-\frac{2 h_{0} \sqrt{k t}}{\sqrt{\pi}} e^{-y^{2} / 4 k t}\right]\right\} \\
= & q_{0}\left[2 \sqrt{\frac{k t}{\pi}} e^{-y^{2} / 4 k t}-y \operatorname{erfc}\left(\frac{y}{2 \sqrt{k t}}\right)\right],
\end{aligned}
$$

which is equivalent to Eq. (21), thus

$$
\lim _{h \rightarrow 0} T_{\text {wet }}(t, y)=T_{\text {dry }}(t, y) .
$$

According to Eqs. (16) and (17), and the results given in Eqs. (32) and (33), the depth of thermal penetration $\delta_{p, h}$ is given by the following equation

$$
\begin{aligned}
& \frac{T_{\text {wet }}\left(t_{c}, \delta_{p, h}\right)-T_{\infty}}{T_{\text {wet }}\left(t_{c}, 0\right)-T_{\infty}} \\
= & \frac{\operatorname{erfc}(\Delta)-e^{\Upsilon^{2}+2 \Delta \Upsilon} \operatorname{erfc}(\Delta+\Upsilon)}{1-e^{\Upsilon^{2}} \operatorname{erfc}(\Upsilon)}=p,
\end{aligned}
$$


where the dimensionless quantities $\Delta$ and $\Upsilon$ are given respectively by Eq. (24) and

$$
\Upsilon=h_{0} \sqrt{k t_{c}} \text {. }
$$

Rewrite now Eq. (35) as

$$
\begin{aligned}
g_{\Upsilon}(\Delta) & =\operatorname{erfc}(\Delta)-e^{\Upsilon^{2}+2 \Delta \Upsilon} \operatorname{erfc}(\Delta+\Upsilon) \\
& =p\left[1-e^{\Upsilon^{2}} \operatorname{erfc}(\Upsilon)\right],
\end{aligned}
$$

thus

$$
\delta_{p, h}=2 \sqrt{k t_{c}} g_{\Upsilon}^{-1}\left(p\left[1-e^{\Upsilon^{2}} \operatorname{erfc}(\Upsilon)\right]\right),
$$

where the inverse function $g_{\Upsilon}^{-1}$ can be computed numerically very easily by using Newton-Raphson's method for root searching. Note that since Eq. (34) holds, $g_{0}^{-1}=g^{-1}$. Notice also that now $\delta_{p, h}$ depends also on the heat transfer coefficient $h$ of the coolant due to Eq. (36).

\section{Two-dimensional approach}

When the heat source starts acting over the surface of the workpiece, the temperature field $T\left(t, x^{\prime}, y^{\prime}\right)$ evolves asymptotically in time $(t \rightarrow \infty)$ to the stationary regime $T\left(x^{\prime}, y^{\prime}\right)$. However, in practice, we can define a relaxation time $t^{*}$ for which $T\left(t, x^{\prime}, y^{\prime}\right) \approx T\left(x^{\prime}, y^{\prime}\right)$, when $t>t^{*}$. In fact, $t^{*}$ will depend on the point of the workpiece $\left(x^{\prime}, y^{\prime}\right)$ considered. In order to avoid thermal damage, the most important point of the workpiece is the one at which maximum temperature $T_{\max }$ is located. According to Ref. [19], $T_{\max }$ is reached asymptotically in the stationary regime on the workpiece surface. For details about the calculation of $T_{\max }$, see Ref. [20] for dry grinding, and Ref. [21] for the wet case. Usually $t^{*}$ is very short, so that during most of the time of the grinding process, the heat transfer occurs very close to the stationary regime. Therefore, we will consider the temperature field in the stationary regime, and for simplicity, we will express it in dimensionless variables (see Table 3), by using the notation $\mathcal{T}(X, Y)$. Also, rewriting the temperature field in dimensionless variables, we can identify the significant parameters on which this heat transfer problem depends: namely, the Peclet number (denoted by Pe), which essentially takes into account the relative movement of the heat source with respect to the workpiece; and the Biot number (denoted by $\mathrm{Bi}$ ), which takes into account the cooling effect of the lubricant.

In this Section, we will collect the results given in the literature regarding the temperature field in the stationary regime for the heat flux profiles considered in this article, i.e. constant, linear, triangular and parabolic, both for dry and wet grinding. From these results, we will describe how to calculate the depth of thermal penetration. 


\begin{tabular}{|c|c|c|}
\hline Meaning & Symbol & Formula \\
\hline Dimensionless abscissa & $X$ & $\frac{v_{d} x^{\prime}}{2 k}$ \\
Dimensionless ordinate & $Y$ & $\frac{v_{d} y^{\prime}}{2 k}$ \\
Dimensionless time & $\tau$ & $\frac{v_{d} \sqrt{t}}{2 \sqrt{k}}$ \\
Peclet number & $\mathrm{Pe}$ & $\frac{v_{d} \ell}{2 k}$ \\
Biot number & $\mathrm{Bi}$ & $\frac{2 h k}{v_{d} k_{0}}$ \\
Dimensionless temperature & $\mathcal{T}$ & $\frac{\pi k_{0} v_{d}\left(T-T_{\infty}\right)}{2 q k}$ \\
\hline
\end{tabular}

Table 3 Dimensionless quantities.

4.1 Temperature field in the stationary regime

\subsubsection{Dry grinding}

For the dry case, according to Ref. [2,22], we have a general expression for the stationary regime

$$
\begin{aligned}
& \mathcal{T}_{\text {dry }}(X, Y) \\
= & \int_{X-L}^{X+L} f(s[X-w]) e^{-w} K_{0}\left(\sqrt{w^{2}+Y^{2}}\right) d w,
\end{aligned}
$$

where remember that $f$ is the dimensionless heat flux profile (see Table 2). It is worth noting that Eq. (38) is given in a slight different way from Ref. [22] because in the latter, the motion of the workpiece is in the opposite direction to the one shown in Fig. 1.

On the surface (i.e. $Y=0$ ), the integral given in Eq. (38) can be calculated for the heat flux profiles considered here (see Ref. [22]). In general, we will use the superscript ${ }^{(k)}$ to indicate the type of heat flux profile within the contact length. Thereby, for the constant case, we have

$$
\mathcal{T}_{\text {dry }}^{(0)}(X, 0)=\left.\operatorname{Jg}_{0}(u)\right|_{u=-X-\mathrm{Pe}} ^{-X+\mathrm{Pe}},
$$

where we have defined the function $\forall n=0,1,2, \ldots$

$$
\begin{aligned}
& \operatorname{Jg}_{n}(x) \\
= & \int_{0}^{x} u^{n} e^{u} K_{0}(|u|) d u \\
= & \left\{\begin{array}{cc}
e^{x} x^{n+1}\left\{K_{0}(|x|) \Psi_{n}(x)+K_{1}(|x|) \Phi_{n}(x)\right\}, & x \neq 0 \\
0 & x=0
\end{array}\right.
\end{aligned}
$$


and we have set the following polynomials in $1 / x$,

$$
\begin{aligned}
& \Psi_{n}(x) \\
& =\frac{1}{n+1}\left[1-\frac{n}{2 n+1}{ }_{3} F_{1}\left(\begin{array}{c}
1,1-n,-1-n \\
\frac{1}{2}-n
\end{array} ; \frac{1}{2 x}\right)\right], \\
& \Phi_{n}(x)=\frac{\operatorname{sgn}(x)}{2 n+1}{ }_{3} F_{1}\left(\begin{array}{c}
1,-n,-n \\
-n+\frac{1}{2}
\end{array} ; \frac{1}{2 x}\right) .
\end{aligned}
$$

For a linear heat flux profile, we have

$$
\mathcal{T}_{\text {dry }}^{(1)}(X, 0)=\frac{1}{\mathrm{Pe}}\left[(\mathrm{Pe}+X) \mathrm{Jg}_{0}(u)+\mathrm{Jg}_{1}(u)\right]_{u=-X-\mathrm{Pe}}^{-X+\mathrm{Pe}} .
$$

In the case of a triangular heat flux profile,

$$
\begin{aligned}
& \mathcal{T}_{\text {dry }}^{(\Delta)}(X, 0) \\
= & 2\left(\frac{\mathcal{H}_{\mathrm{Pe}}(X)}{1-\lambda}+\frac{\mathcal{H}_{-\mathrm{Pe}}(X)}{1+\lambda}+\frac{2 \lambda}{\lambda^{2}-1} \mathcal{H}_{\lambda \mathrm{Pe}}(X)\right),
\end{aligned}
$$

where we have set

$$
\mathcal{H}_{\Lambda}(X)=\operatorname{Jg}_{0}(\Lambda-X)\left(1-\frac{X}{\Lambda}\right)-\frac{1}{\Lambda} \operatorname{Jg}_{1}(\Lambda-X) .
$$

Finally, for the parabolic case,

$$
\mathcal{T}_{\text {dry }}^{(2)}(X, 0)=\left.\frac{3}{4 \mathrm{Pe}^{2}} \sum_{n=0}^{2} c_{n}(X) \mathrm{Jg}_{n}(u)\right|_{u=-X-\mathrm{Pe}} ^{-X+\mathrm{Pe}},
$$

where

$$
\begin{aligned}
& c_{0}(X)=(\mathrm{Pe}+X)^{2}, \\
& c_{1}(X)=2(\mathrm{Pe}+X), \\
& c_{2}(X)=1 .
\end{aligned}
$$

\subsubsection{Wet grinding}

For the wet case, i.e. $b\left(t, x^{\prime}\right)=h$, according to Ref. [21], the boundary-value problem given in Eqs. (2), (5) and (4) can be solved in integral form as,

$$
\mathcal{T}_{\text {wet }}^{(k)}(\tau, X, Y)=\int_{0}^{\tau} B_{\mathrm{Bi}}(Y, w) P_{\mathrm{Pe}}^{(k)}(X, w) d w,
$$

where the $B_{\mathrm{Bi}}(Y, w)$ function takes into account the ordinate $Y$ and the effect of the coolant,

$$
\begin{aligned}
& B_{\mathrm{Bi}}(Y, w) \\
= & \exp \left(\frac{-Y^{2}}{4 w^{2}}\right)-\sqrt{\pi} \operatorname{Bi} w e^{\mathrm{Bi} Y+\mathrm{Bi}^{2} w^{2}} \operatorname{erfc}\left(\operatorname{Bi} w+\frac{Y}{2 w}\right),
\end{aligned}
$$


and the $P_{\mathrm{Pe}}^{(k)}(X, w)$ function takes into account the abscissa $X$ and the type of heat flux profile considered (i.e. superscript $\left.{ }^{(k)}\right)$. For instance, considering a constant heat flux profile, we have,

$$
P_{\mathrm{Pe}}^{(0)}(X, w)=\sqrt{\pi}\left[\operatorname{erf}\left(\frac{u}{2 w}+w\right)\right]_{u=X-\mathrm{Pe}}^{X+\mathrm{Pe}},
$$

which has been given first in Ref. [23]. The linear case, first given in Ref. [13], reads as

$$
\begin{aligned}
P_{\mathrm{Pe}}^{(1)}(X, w)= & \frac{1}{\mathrm{Pe}}\left[\sqrt{\pi}\left(X+\mathrm{Pe}+2 w^{2}\right) \operatorname{erf}\left(\frac{u}{2 w}+w\right)\right. \\
& \left.+2 w \exp \left(-\left[\frac{u}{2 w}+w\right]^{2}\right)\right]_{u=X-\mathrm{Pe}}^{X+\mathrm{Pe}}
\end{aligned}
$$

For the triangular profile, it turns out that

$$
\begin{aligned}
& P_{\mathrm{Pe}}^{(\Delta)}(X, w) \\
= & \frac{4 w}{\operatorname{Pe}}\left[\frac{\varphi\left(\frac{X+\mathrm{Pe}}{2 w}+w\right)}{\lambda+1}+\frac{2 \varphi\left(\frac{X-\lambda \mathrm{Pe}}{2 w}+w\right)}{\lambda^{2}-1}-\frac{\varphi\left(\frac{X-\mathrm{Pe}}{2 w}+w\right)}{\lambda-1}\right],
\end{aligned}
$$

where we have set

$$
\varphi(x)=\sqrt{\pi} x \operatorname{erf}(x)+\exp \left(-x^{2}\right) .
$$

Finally, for a parabolic heat flux profile, we have

$$
\begin{aligned}
& P_{\mathrm{Pe}}^{(2)}(X, w) \\
= & \frac{3}{4 \mathrm{Pe}^{2}}\left[\sqrt{\pi}\left(2 w^{2}+\left(\mathrm{Pe}+2 w^{2}+X\right)^{2}\right) \operatorname{erf}\left(\frac{u}{2 w}+w\right)\right. \\
& \left.+2 w\left(2 \mathrm{Pe}+2 X+2 w^{2}-u\right) \exp \left(-\left[\frac{u}{2 w}+w\right]^{2}\right)\right]_{u=X-\mathrm{Pe}}^{X+\mathrm{Pe}}
\end{aligned}
$$

From Eq. (47), it is clear that in the stationary regime, we have to take the limit $\tau \rightarrow \infty$, that is to say,

$$
\mathcal{T}_{\text {wet }}^{(k)}(X, Y)=\int_{0}^{\infty} B_{\mathrm{Bi}}(Y, w) P_{\mathrm{Pe}}^{(k)}(X, w) d w .
$$

From a numerical point of view, instead of computing Eq. (53), it is much faster to approximate it taking into account the dimensionless relaxation time $\tau^{*}$ as follows

$$
\mathcal{T}_{\text {wet }}^{(k)}(X, Y) \approx \int_{0}^{\tau^{*}} B_{\mathrm{Bi}}(Y, w) P_{\mathrm{Pe}}^{(k)}(X, w) d w,
$$

since the temperature field hardly evolves for $\tau>\tau^{*}$. It should be noted that the numerical integration of Eq. (54) is quite efficient if we use the double 
exponential strategy, Ref. [24]. Nonetheless, the particular case of the temperature field on the surface $(Y=0)$ for a constant heat flux profile is given in terms of a series that can be computed very rapidly, Ref. [25],

$$
\mathcal{T}_{\text {wet }}^{(0)}(X, 0)=\left.\sqrt{\pi} \sum_{n=0}^{\infty} \frac{(-\mathrm{Bi} / \sqrt{2})^{n}}{\Gamma\left(\frac{n+1}{2}\right)} \mathrm{Yu}_{n / 2}(u)\right|_{u=-X-\mathrm{Pe}} ^{-X+\mathrm{Pe}},
$$

where

$$
\begin{aligned}
& \mathrm{Yu}_{\nu}(x)= \\
& \left\{\begin{array}{cc}
\frac{x|x|^{\nu} e^{x}}{2 \nu+1}\left[K_{\nu}(|x|)+\operatorname{sign}(x) K_{\nu+1}(|x|)\right] & x \neq 0 \\
0 & x=0
\end{array}\right.
\end{aligned}
$$

and where $K_{\nu}(x)$ denotes the $\nu$-th order modified Bessel function of the second kind (see Ref. [26, Chap. 51]). On the one hand, it is worth noting that in Ref. [25] the direction of the wheel is just the opposite of the one considered in Fig. 1, hence the abscissa has been changed to $-X$. On the other hand, the series given in Eq. (55) does not converge for high Biot numbers, i.e. $\mathrm{Bi}>1$. However, for $\mathrm{Bi}>1$, we can still using the general formula given in Eq. (54).

\subsection{Depth of thermal penetration}

The contour $Y(X)$ of the temperature field for a fixed dimensionless temperature $\mathcal{T}_{p}$ (see Table 3 ) is given in implicit form as

$$
F(X, Y)=\mathcal{T}(X, Y)-\mathcal{T}_{p}=0
$$

In order to define a depth of thermal penetration similar to the onedimensional case, i.e. (16), we are interested in the contour $Y(X)$ for which $\mathcal{T}_{p}$ is a fraction $p$ of the dimensionless maximum temperature $\mathcal{T}_{\max }$ (which occurs on the surface in the stationary regime, as aforementioned),

$$
\mathcal{T}_{p}=p \mathcal{T}_{\max }
$$

Therefore, if $\left(X_{p}, Y_{p}\right)$ is the point of the contour $Y(X)$ for which $Y_{p}\left(X_{p}\right)$ reaches the global maximum, then $Y_{p}$ is just the dimensionless depth of thermal penetration for grinding (see Fig. 3).

On the one hand, we are going to provide in this Section the equations for searching the maximum depth for which the workpiece reaches $\mathcal{T}_{p}$, according to the type of heat flux profile (constant, linear, triangular and parabolic) and whether we consider also dry or wet grinding.

On the other hand, in order to set the most adequate $p$ value in surface grinding, we can consider the following cases. If burnout of the workpiece occurs, we could take $\mathcal{T}_{p}=\mathcal{T}_{\text {burn }}$, where $\mathcal{T}_{\text {burn }}$ is the dimensionless temperature correponding to the phase change temperature of the workpiece material. Thereby, in this case, $\delta_{p}$ will coincide with the depth of the burnt layer on the 


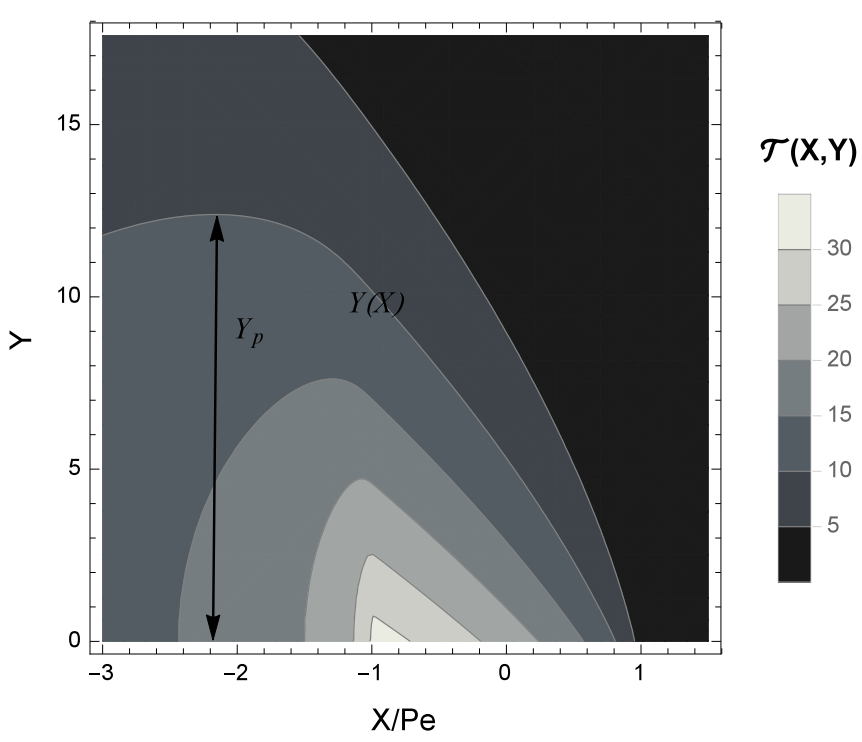

Fig. 3 Dimensionless depth of thermal penetration for a two-dimensional model in grinding.

workpiece surface. Anyway, other types of thermal damage of the workpiece may occur in the workpiece, such as tempering in steel, which produces a hardness ditribution beneath the ground surface. In this case, we can set $\mathcal{T}_{p}=$ $\mathcal{T}_{\text {tempering }}$, where $\mathcal{T}_{\text {tempering }}$ is the dimensionless temperature corresponding to the tempering temperature of the steel.

If thermal damage does not occur, we could set $p$ in a similar way as in the definition of the half-life of many decay physics processes, so that $p=0.5$. As an alternative to half-life, the mean life is defined in exponential decay processes, being $p=1 / e \approx 0.37$. In any one of the latter cases, $\delta_{p}$ will provide a characteristic depth of the temperature field in grinding. However, the authors have checked that $p=0.1$ is a fair value in order to set the domain in the graphical plot of the temperature field.

\subsubsection{Dry grinding}

According to Eq. (38) and Table 2, Eq. (57) reads as follows for a constant heat flux profile,

$$
F_{\text {dry }}^{(0)}(X, Y)=\int_{X-\mathrm{Pe}}^{X+\mathrm{Pe}} e^{-w} K_{0}\left(\sqrt{w^{2}+Y^{2}}\right) d w-\mathcal{T}_{p}=0 .
$$

We want to know the maximum depth for which the workpiece reaches the dimensionless temperature $\mathcal{T}_{p}$. For this purpose, let us calculate the critical points $\left(X_{p}, Y_{p}\right)$ of Eq. (59), i.e. the points such that $Y_{p}=Y\left(X_{p}\right)$ and $Y^{\prime}\left(X_{p}\right)=$ 0 . Therefore, let us apply the Leibniz's theorem for differentiation of integrals 
(see Ref. [27, Eqn. 1.5.22]) to Eq. (59), in order to obtain

$$
\begin{aligned}
& 0=\frac{\partial F_{\mathrm{dry}}^{(0)}}{\partial X} \\
= & e^{-(X+\mathrm{Pe})} K_{0}\left(\sqrt{(X+\mathrm{Pe})^{2}+Y_{p}^{2}}\right)-e^{-(X-\mathrm{Pe})} K_{0}\left(\sqrt{(X-\mathrm{Pe})^{2}+Y^{2}}\right) \\
& -Y Y^{\prime} \int_{X-\mathrm{Pe}}^{X+\mathrm{Pe}} e^{-w} \frac{K_{1}\left(\sqrt{w^{2}+Y^{2}}\right)}{\sqrt{w^{2}+Y^{2}}} d w .
\end{aligned}
$$

Imposing now the condition $Y^{\prime}\left(X_{p}\right)=0$ and knowing that $Y_{p}=Y\left(X_{p}\right)$, we have the following system of non-linear equations for $\left(X_{p}, Y_{p}\right)$,

$$
\begin{aligned}
0= & e^{-\left(X_{p}+\mathrm{Pe}\right)} K_{0}\left(\sqrt{\left(X_{p}+\mathrm{Pe}\right)^{2}+Y_{p}^{2}}\right) \\
& -e^{-\left(X_{p}-\mathrm{Pe}\right)} K_{0}\left(\sqrt{\left(X_{p}-\mathrm{Pe}\right)^{2}+Y_{p}^{2}}\right), \\
0= & \int_{X_{p}-\mathrm{Pe}}^{X_{p}+\mathrm{Pe}} e^{-w} K_{0}\left(\sqrt{w^{2}+Y_{p}^{2}}\right) d w-\mathcal{T}_{p} .
\end{aligned}
$$

In order to solve Eqs. (60) and (61) numerically, we need to provide a starting iteration point $\left(X_{0}, Y_{0}\right)$ that converges to the critical point $\left(X_{p}, Y_{p}\right)$. We can find this starting iteration point heuristically as follows. First, let us use the trapezoidal rule,

$$
\int_{a}^{b} f(u) d u \approx \frac{b-a}{2}[f(a)+f(b)],
$$

for approximating (61) as

$$
\begin{aligned}
\frac{\mathcal{T}_{p}}{\mathrm{Pe} \approx} & e^{-\left(X_{p}+\mathrm{Pe}\right)} K_{0}\left(\sqrt{\left(X_{p}+\mathrm{Pe}\right)^{2}+Y_{p}^{2}}\right) \\
& +e^{-\left(X_{p}-\mathrm{Pe}\right)} K_{0}\left(\sqrt{\left(X_{p}-\mathrm{Pe}\right)^{2}+Y_{p}^{2}}\right) .
\end{aligned}
$$

Second, substitute

$$
X_{0}=-\mathrm{Pe},
$$

in Eqs. (60) and (62), to obtain

$$
\begin{aligned}
& K_{0}\left(\left|Y_{0}\right|\right)-e^{2 \mathrm{Pe}} K_{0}\left(\sqrt{4 \mathrm{Pe}^{2}+Y_{0}^{2}}\right)=0, \\
& K_{0}\left(\left|Y_{0}\right|\right)+e^{2 \mathrm{Pe}} K_{0}\left(\sqrt{4 \mathrm{Pe}^{2}+Y_{0}^{2}}\right) \approx \frac{\mathcal{T}_{p}}{\mathrm{Pe}} .
\end{aligned}
$$


Notice that summing up Eqs. (64) and (65) we obtain a simple equation that can replace Eq. (65), hence

$$
\begin{aligned}
K_{0}\left(\left|Y_{0}\right|\right)-e^{2 \mathrm{Pe}} K_{0}\left(\sqrt{4 \mathrm{Pe}^{2}+Y_{0}^{2}}\right) & =0, \\
K_{0}\left(\left|Y_{0}\right|\right) & \approx \frac{\mathcal{T}_{p}}{2 \mathrm{Pe}} .
\end{aligned}
$$

If $\mathrm{Pe} \rightarrow 0$, then Eq. (66) is satisfied asymptotically $\forall Y_{0}$, and Eq. (67) yields

$$
Y_{0} \approx K_{0}^{-1}\left(\frac{\mathcal{T}_{p}}{2 \mathrm{Pe}}\right)
$$

where $K_{0}^{-1}(x)$ denotes the inverse function of the zeroth order modified Bessel function of the second kind.

Also, if $\mathrm{Pe} \rightarrow \infty$, then Eqs. (66) and (67) become

$$
\begin{aligned}
& K_{0}\left(\left|Y_{0}\right|\right) \approx 0, \\
& K_{0}\left(\left|Y_{0}\right|\right) \approx \frac{\mathcal{T}_{p}}{2 \mathrm{Pe}} \approx 0,
\end{aligned}
$$

so that the approximation given in Eq. (68) also works as a good initial iteration point.

Note that since $K_{0}(x)$ is a concave function for positive arguments, it is quite easy to compute its inverse by using Newton-Raphson method. As we will see later on in Section 5 , it turns out that the initial point $\left(X_{0}, Y_{0}\right)$ given in Eqs. (63) and (68) converges most of the times quite well to the critical point $\left(X_{p}, Y_{p}\right)$, not only for a constant heat flux profile, but for others heat flux profiles, such as linear, triangular and parabolic.

Nonetheless, take into account that the non-linear system for others heat flux profiles is different from the one given in Eqs. (60)-(61). For instance, for a linear heat flux profile, according to Eq. (38) and Table 2, Eq. (57) reads for the critical point $\left(X_{p}, Y_{p}\right)$ as

$$
\begin{aligned}
& F_{\text {dry }}^{(1)}\left(X_{p}, Y_{p}\right) \\
= & \frac{1}{\mathrm{Pe}} \int_{X_{p}-\mathrm{Pe}}^{X_{p}+\mathrm{Pe}}\left(\mathrm{Pe}+X_{p}-w\right) e^{-w} K_{0}\left(\sqrt{w^{2}+Y_{p}^{2}}\right) d w-\mathcal{T}_{p} \\
= & 0 .
\end{aligned}
$$

Applying the Leibniz's theorem for differentiation of integrals as explained above, we arrive at the second equation for the critical point,

$$
\begin{aligned}
& \int_{X_{p}-\mathrm{Pe}}^{X_{p}+\mathrm{Pe}} e^{-w} K_{0}\left(\sqrt{w^{2}+Y_{p}^{2}}\right) d w \\
= & 2 \operatorname{Pe} e^{-\left(X_{p}-\mathrm{Pe}\right)} K_{0}\left(\sqrt{\left(X_{p}-\mathrm{Pe}\right)^{2}+Y_{p}^{2}}\right) .
\end{aligned}
$$


Similarly, for a triangular heat flux profile, we obtain

$$
\begin{aligned}
0= & F_{\mathrm{dry}}^{(\Delta)}\left(X_{p}, Y_{p}\right) \\
= & \mathcal{T}_{p}-2 \int_{X_{p}-\mathrm{Pe}}^{X_{p}+\mathrm{Pe}} e^{-w} K_{0}\left(\sqrt{w^{2}+Y_{p}^{2}}\right) \\
& \times\left(1-\frac{w-X_{p}+\lambda \mathrm{Pe}}{\operatorname{Pe}\left[\lambda+\operatorname{sign}\left(w-X_{p}+\lambda \mathrm{Pe}\right)\right]}\right) d w
\end{aligned}
$$

Nevertheless, in order to apply the Leibniz's theorem for differentiation of integrals, we can rewrite $F_{\text {dry }}^{(\Delta)}(X, Y)$ as follows,

$$
\begin{aligned}
& F_{\mathrm{dry}}^{(\Delta)}(X, Y) \\
= & \frac{2}{\lambda+1} \int_{X-\lambda \mathrm{Pe}}^{X+\mathrm{Pe}}\left(\frac{X-w}{\mathrm{Pe}}+1\right) e^{-w} K_{0}\left(\sqrt{w^{2}+Y^{2}}\right) d w \\
& +\frac{2}{\lambda-1} \int_{X-\mathrm{Pe}}^{X-\lambda \mathrm{Pe}}\left(\frac{X-w}{\mathrm{Pe}}-1\right) e^{-w} K_{0}\left(\sqrt{w^{2}+Y^{2}}\right) d w .
\end{aligned}
$$

After some algebra, we arrive at

$$
\begin{aligned}
& \frac{\partial F_{\mathrm{dry}}^{(\Delta)}}{\partial X} \\
= & \frac{2}{\operatorname{Pe}}\left\{\int_{X-\lambda \mathrm{Pe}}^{X+\mathrm{Pe}} \frac{e^{-w} K_{0}\left(\sqrt{w^{2}+Y^{2}}\right)}{\lambda+1} d w\right. \\
& \left.+\int_{X-\mathrm{Pe}}^{X-\lambda \mathrm{Pe}} \frac{e^{-w} K_{0}\left(\sqrt{w^{2}+Y^{2}}\right)}{\lambda-1} d w\right\},
\end{aligned}
$$

that can be recast and particularized to the critical point $\left(X_{p}, Y_{p}\right)$ as

$$
\int_{X_{p}-\mathrm{Pe}}^{X_{p}+\mathrm{Pe}} \frac{e^{-w} K_{0}\left(\sqrt{w^{2}+Y_{p}^{2}}\right)}{\lambda+\operatorname{sign}\left(w-X_{p}+\lambda \mathrm{Pe}\right)} d w=0 .
$$

Finally, the system of non-linear equations for a parabolic heat flux profile is given by

$$
\begin{aligned}
& 0=F_{\mathrm{dry}}^{(2)}\left(X_{p}, Y_{p}\right) \\
= & \frac{3}{4 \mathrm{Pe}^{2}} \int_{X-\mathrm{Pe}}^{X+\mathrm{Pe}}(\mathrm{Pe}+X-w)^{2} e^{-w} K_{0}\left(\sqrt{w^{2}+Y^{2}}\right) d w-\mathcal{T}_{p},
\end{aligned}
$$

and

$$
\begin{aligned}
& \int_{X_{p}-\mathrm{Pe}}^{X_{p}+\mathrm{Pe}}\left(\mathrm{Pe}+X_{p}-w\right) e^{-w} K_{0}\left(\sqrt{w^{2}+Y_{p}^{2}}\right) d w \\
= & 2 \mathrm{Pe}^{2} e^{-\left(X_{p}-\mathrm{Pe}\right)} K_{0}\left(\sqrt{\left(X_{p}-\mathrm{Pe}\right)^{2}+Y_{p}^{2}}\right) .
\end{aligned}
$$




\subsubsection{Wet grinding}

Considering a constant heat flux profile, according to Eqs. (54) and (48), Eq. (57) for the critical point $\left(X_{p}, Y_{p}\right)$ reads as

$$
\begin{aligned}
& F_{\mathrm{wet}}^{(0)}\left(X_{p}, Y_{p}\right) \\
\approx & \int_{0}^{\tau^{*}} B_{\mathrm{Bi}}\left(Y_{p}, w\right) P_{\mathrm{Pe}}^{(0)}\left(X_{p}, w\right) d w-\mathcal{T}_{p} \\
= & 0
\end{aligned}
$$

where $P_{\mathrm{Pe}}^{(0)}(X, w)$ is given in Eq. (48). Now, differentiating $F_{\text {wet }}^{(0)}$ with respect to $X$, the second equation for the critical point $\left(X_{p}, Y_{p}\right)$ is

$$
\int_{0}^{\infty} B_{\mathrm{Bi}}\left(Y_{p}, w\right) \hat{P}_{\mathrm{Pe}}^{(0)}\left(X_{p}, w\right) d w=0
$$

where

$$
\hat{P}_{\mathrm{Pe}}^{(0)}(X, w)=\frac{1}{w}\left[\exp \left(-\left[\frac{u}{2 w}+w\right]^{2}\right)\right]_{u=X-\mathrm{Pe}}^{X+\mathrm{Pe}} .
$$

As aforementioned in Eq. (54), it is worth noting that the infinite upper limit in Eq. (75) has been replaced by the relaxation time $\tau^{*}$ in order to speed up the computation of the integral. Nevertheless, this is not the case of (76), since the behavior of the integrand is different. This remark can be applied for the non-linear equations of the others heat flux profiles presented below.

For a linear heat flux profile, from Eqs. (54) and (49), we have the following system of non-linear equations for the critical point $\left(X_{p}, Y_{p}\right)$ :

$$
\begin{aligned}
& F_{\text {wet }}^{(1)}\left(X_{p}, Y_{p}\right) \\
\approx & \int_{0}^{\tau^{*}} B_{\mathrm{Bi}}\left(Y_{p}, w\right) P_{\mathrm{Pe}}^{(1)}\left(X_{p}, w\right) d w-\mathcal{T}_{p}=0,
\end{aligned}
$$

where $P_{\mathrm{Pe}}^{(1)}(X, w)$ is given in Eq. (49); and

$$
\int_{0}^{\infty} B_{\mathrm{Bi}}\left(Y_{p}, w\right) \hat{P}_{\mathrm{Pe}}^{(1)}\left(X_{p}, w\right) d w=0
$$

where

$$
\begin{aligned}
& \hat{P}_{\mathrm{Pe}}^{(1)}(X, w) \\
= & \left.\sqrt{\pi} \operatorname{erf}\left(\frac{u}{2 w}+w\right)\right|_{u=X-\mathrm{Pe}} ^{X+\mathrm{Pe}} \\
& -\frac{2 \operatorname{Pe}}{w} \exp \left(-\left[\frac{X-\mathrm{Pe}}{2 w}+w\right]^{2}\right) .
\end{aligned}
$$


Consider now a triangular heat flux profile, so that

$$
\begin{aligned}
& F_{\text {wet }}^{(\Delta)}\left(X_{p}, Y_{p}\right) \\
\approx & \int_{0}^{\tau^{*}} B_{\mathrm{Bi}}\left(Y_{p}, w\right) P_{\mathrm{Pe}}^{(\Delta)}\left(X_{p}, w\right) d w-\mathcal{T}_{p}=0,
\end{aligned}
$$

where $P_{\mathrm{Pe}}^{(\Delta)}(X, w)$ is given in Eq. (50); and

$$
\int_{0}^{\infty} B_{\mathrm{Bi}}\left(Y_{p}, w\right) \hat{P}_{\mathrm{Pe}}^{(\Delta)}\left(X_{p}, w\right) d w=0
$$

where

$$
\begin{aligned}
& \hat{P}_{\mathrm{Pe}}^{(\Delta)}(X, w) \\
= & \frac{\operatorname{erf}\left(\frac{X+\mathrm{Pe}}{2 w}+w\right)}{\lambda+1}+\frac{2 \operatorname{erf}\left(\frac{X-\lambda \mathrm{Pe}}{2 w}+w\right)}{\lambda^{2}-1}-\frac{\operatorname{erf}\left(\frac{X-\mathrm{Pe}}{2 w}+w\right)}{\lambda-1} .
\end{aligned}
$$

Finally, for a parabolic heat flux profile, we have

$$
\begin{aligned}
& F_{\text {wet }}^{(2)}\left(X_{p}, Y_{p}\right) \\
\approx & \int_{0}^{\tau^{*}} B_{\mathrm{Bi}}\left(Y_{p}, w\right) P_{\mathrm{Pe}}^{(2)}\left(X_{p}, w\right) d w-\mathcal{T}_{p} \\
= & 0
\end{aligned}
$$

where $P_{\mathrm{Pe}}^{(2)}(X, w)$ is given in Eq. (52); and

$$
\int_{0}^{\infty} B_{\mathrm{Bi}}\left(Y_{p}, w\right) \hat{P}_{\mathrm{Pe}}^{(2)}\left(X_{p}, w\right) d w=0
$$

where

$$
\begin{aligned}
& \hat{P}_{\mathrm{Pe}}^{(2)}(X, w) \\
= & 2 w\left[\exp \left(-\left[\frac{X+\mathrm{Pe}}{2 w}+w\right]^{2}\right)\right. \\
& \left.-\exp \left(-\left[\frac{X-\mathrm{Pe}}{2 w}+w\right]^{2}\right)\left(\frac{\mathrm{Pe}^{2}}{w^{2}}+1\right)\right] \\
& +\sqrt{\pi}\left(\mathrm{Pe}+2 w^{2}+X\right)\left[\operatorname{erf}\left(\frac{u}{2 w}+w\right)\right]_{u=X-\mathrm{Pe}}^{X+\mathrm{Pe}}
\end{aligned} .
$$




\begin{tabular}{|c|c|c|c|c|}
\cline { 3 - 5 } \multicolumn{2}{c|}{} & Data 1 & Data 2 & Data 3 \\
\hline \hline \multirow{3}{*}{ Workpiece } & Material & VT20 & Carbon Steel & Sapphire \\
\cline { 2 - 5 } & $k_{0}$ & 13 & 60.5 & 46 \\
\cline { 2 - 5 } & $k$ & $4.23 \times 10^{-6}$ & $1.77 \times 10^{-5}$ & $1.51 \times 10^{-5}$ \\
\hline \hline \multirow{3}{*}{$\begin{array}{c}\text { Grinding } \\
\text { Regime }\end{array}$} & $2 \ell$ & $2.66 \times 10^{-3}$ & $1.4 \times 10^{-2}$ & $2.5 \times 10^{-3}$ \\
\cline { 2 - 5 } & $q$ & $5.89 \times 10^{7}$ & $1.4 \times 10^{7}$ & $1.8 \times 10^{7}$ \\
\cline { 2 - 5 } & $v_{d}$ & 0.53 & $3.3 \times 10^{-2}$ & 0.33 \\
\cline { 2 - 5 } & $T_{\infty}$ & 300 & 300 & 300 \\
\hline \multirow{2}{*}{$\begin{array}{c}\text { Dimensionless } \\
\text { Parameters }\end{array}$} & $\mathrm{Pe}$ & $2.729 \times 10^{5}$ & $1.3 \times 10^{5}$ & $4.1 \times 10^{5}$ \\
\cline { 2 - 5 } & $\mathrm{Bi}$ & 83.4 & 0.651 & 13.6 \\
\hline
\end{tabular}

Table 4 Simulation parameters in SI units.

\section{Numerical results}

Table 4 shows three sets of parameters (in SI units) for the numerical simulations. Data set 1 considers a titanium alloy VT20 workpiece, whose thermal properties are given in Ref. [28]. The grinding regime for this simulation can be found in Ref. [10]. Data sets 2 and 3 consider carbon steel and aluminum oxide (sapphire) as workpiece material, whose thermal properties and grinding regimes are given in Ref. [29]. These data sets have been chosen in order to have a wide range of Peclet number values and different Biot number values.

For the computation of the exact depth of thermal penetration shown in Figs. 4-9, we seek first a value $p$ for which the initial seed given in Eqs. (63) and (68) converges properly to the solution of the non-linear equations posed in Section 4.2 for the different heat flux profiles considered. We use the latter solution as the initial seed for nearby values of $p$, and iteratively the depth of thermal penetration is calculated for the range presented in Figs. 4-9, i.e. $0.05 \leq p \leq 1$.

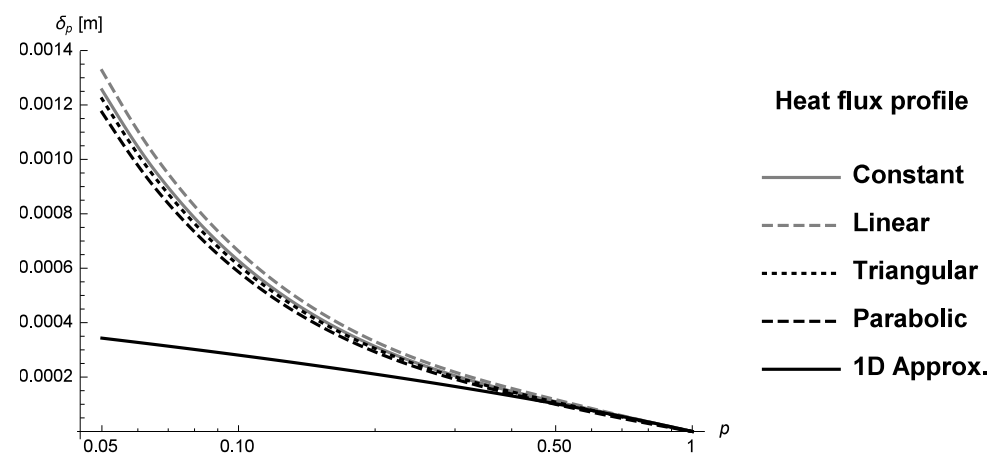

Fig. 4 Depth of thermal penetration for data set 1 in dry grinding. 


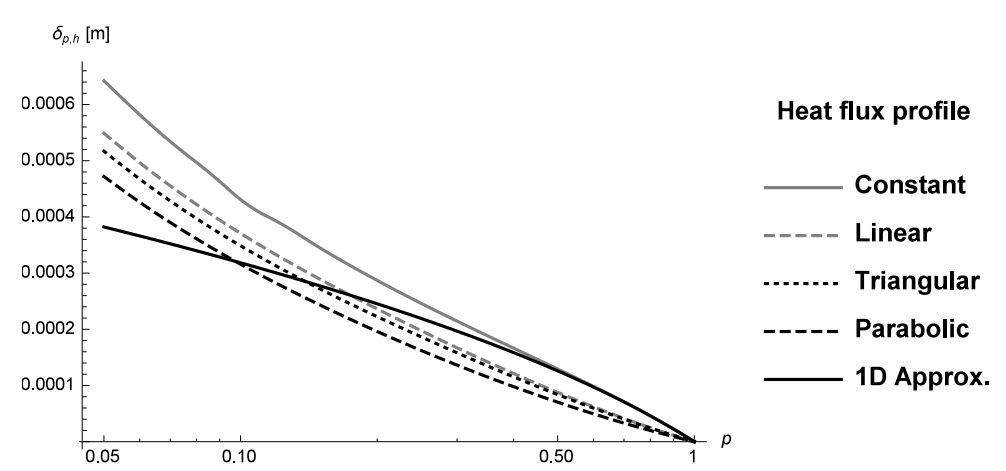

Fig. 5 Depth of thermal penetration for data set 1 in wet grinding.

As a common feature of all graphs in dry grinding, the one-dimensional approximation provides an accurate value for $0.4 \leq p \leq 1$, regardless the heat flux profile considered. Physically speaking, this is to be expected since nearby the heat source, the heat transfer is conducted mainly on the $y$-axis direction during time $t_{c}$. In fact, a point on the surface is heated up by the heat source just time $t_{c}$. Remember that the boundary conditions for the one-dimensional approach, Eqs. (14) and (15), have been approximated taking into account this characteristic time, and thereby the depth of thermal penetration $\delta_{p}$ have been defined for $t_{c}$ as well, as indicated in Eq. (17). Therefore, the one-dimensional approach coincide with the two-dimensional one on the workpiece surface, i.e. for $p=1$ according Eq. (16), as it is seen in all graphs.

In the case of wet grinding, the one-dimensional approach provides much better accuracy for a constant heat flux profile than for any other heat flux profile. This is consistent with the assumption performed in the boundary condition given in Eq. (10) for the one-dimensional approach. However, for data set 2, the one-dimensional approximation deviates from the exact result for a constant heat flux profile, since the Peclet number is smaller than in the other cases. This is consistent with the approximation given in Eq. (13).

Also, the depth of thermal penetration depends more on the heat flux profile in wet grinding than in dry grinding. In contrast, the growth of the depth of thermal penetration is steeper for small $p$ in dry grinding than in wet grinding.

Finally, we have compared the one- and two-dimensional approaches of the depth of thermal penetration to the temperature field given in Ref. [30], computed by using a three-dimensional FEM analysis. This FEM analysis considers a TC4 titanium alloy as workpiece material, and a linear heat flux profile in dry grinding. Table 5 shows the input parameters for this simulation as well as the maximum temperature $T_{\max }(y)$ at given depths $y$ beneath the workpiece surface. 


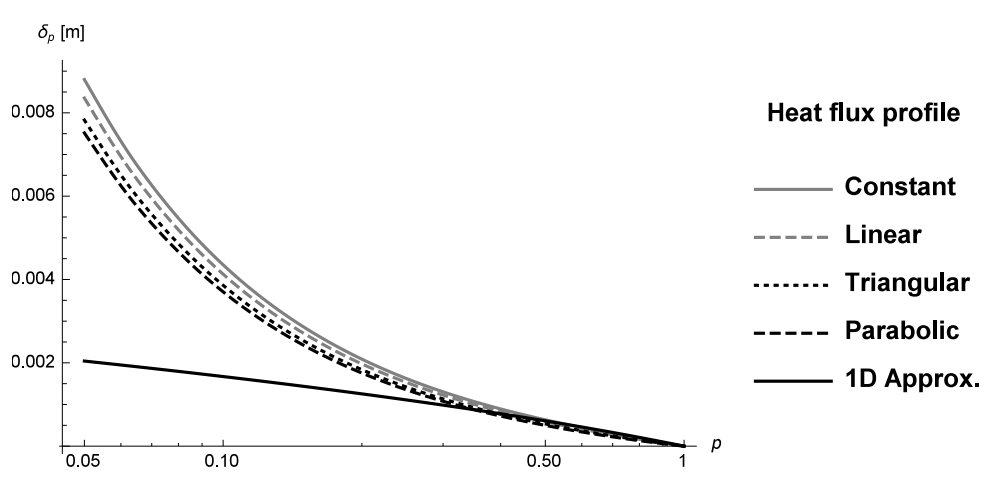

Fig. 6 Depth of thermal penetration for data set 2 in dry grinding.

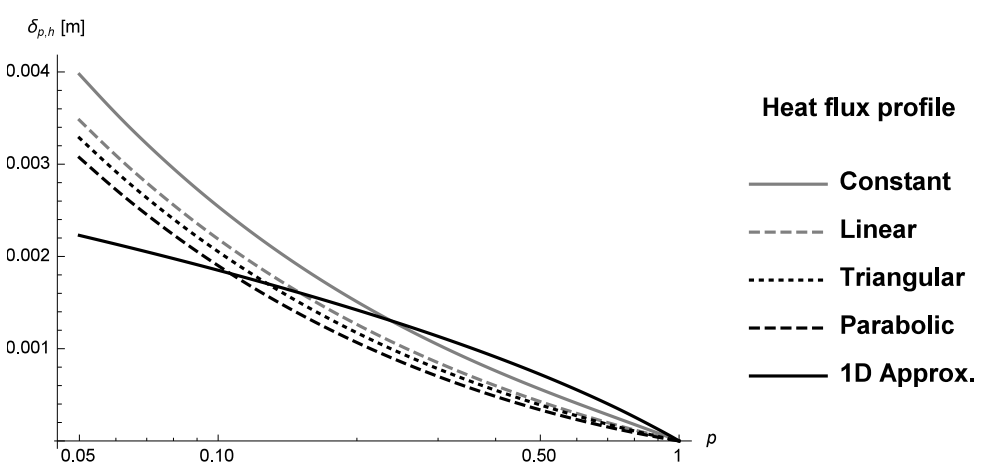

Fig. 7 Depth of thermal penetration for data set 2 in wet grinding.

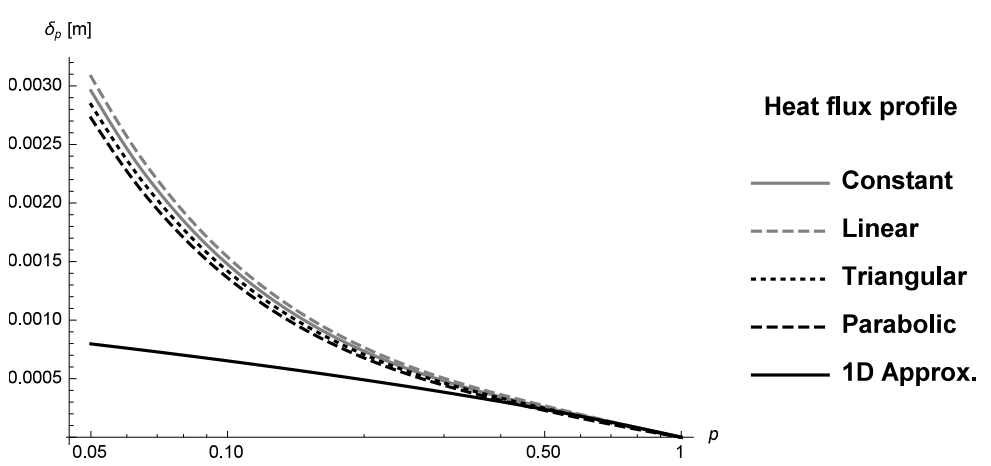

Fig. 8 Depth of thermal penetration for data set 3 in dry grinding.

According to Eq. (16), the $p$ values of Fig. 10 have been calculated as

$$
p=\frac{T_{\max }(y)-T_{\infty}}{T_{\max }(0)-T_{\infty}},
$$

and then, we have used Eq. (26) to calculate $\delta_{p}$ for the one-dimensional approach, and Eqs. (69)-(70) for the two-dimensional approach. These $\delta_{p}$ values 


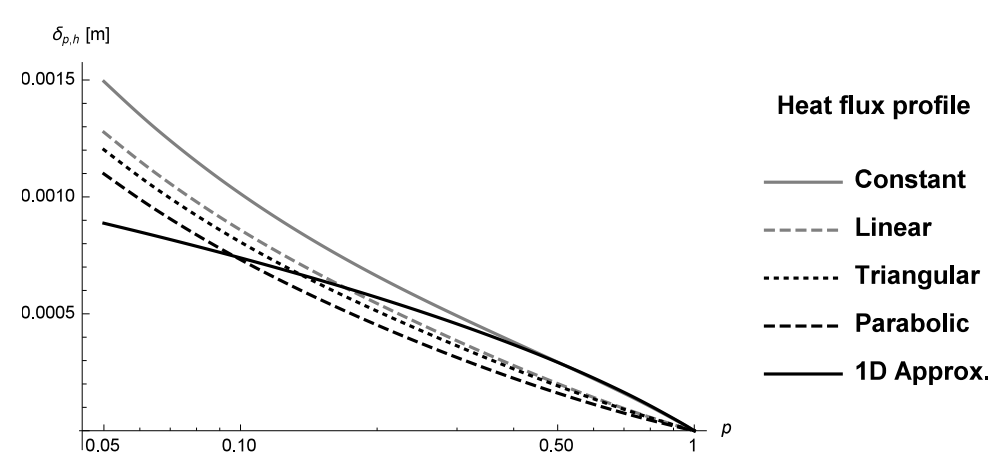

Fig. 9 Depth of thermal penetration for data set 3 in wet grinding.

\begin{tabular}{|c|c||c||c|c|}
\cline { 3 - 5 } \multicolumn{2}{c|}{} & Data & $T_{\max }$ & $y$ \\
\hline \hline \multirow{3}{*}{ Workpiece } & Material & TC4 & 611.4 & 0 \\
\cline { 2 - 5 } & $k_{0}$ & 7.955 & 572.5 & $1 \times 10^{-4}$ \\
\cline { 2 - 5 } & $k$ & $3.36 \times 10^{-6}$ & 539.9 & $2 \times 10^{-4}$ \\
\hline \multirow{3}{*}{$\begin{array}{c}\text { Grinding } \\
\text { Regime }\end{array}$} & $2 \ell$ & $2.83 \times 10^{-3}$ & 512.0 & $3 \times 10^{-4}$ \\
\cline { 2 - 5 } & $q$ & $3.14 \times 10^{6}$ & 487.9 & $4 \times 10^{-4}$ \\
\cline { 2 - 5 } & $v_{d}$ & $1.66 \times 10^{-2}$ & 467.2 & $5 \times 10^{-4}$ \\
\cline { 2 - 5 } & $T_{\infty}$ & 288 & 449.4 & $6 \times 10^{-4}$ \\
\cline { 2 - 5 } & $h$ & 0 & 434.0 & $7 \times 10^{-4}$ \\
\hline Dimensionless & $\mathrm{Pe}$ & 3.51 & 420.8 & $8 \times 10^{-4}$ \\
\cline { 2 - 5 } Parameters & $\mathrm{Bi}$ & 0 & 409.5 & $9 \times 10^{-4}$ \\
\hline
\end{tabular}

Table 5 Data extracted from Wang et al. [30] in SI units.

are compared to the $y$ values listed in Table 5 and presented in Fig. 10. We can see that the two-dimensional approach fits better to the three-dimensional FEM data, but both approaches are very good for $0.6 \leq p \leq 1$.

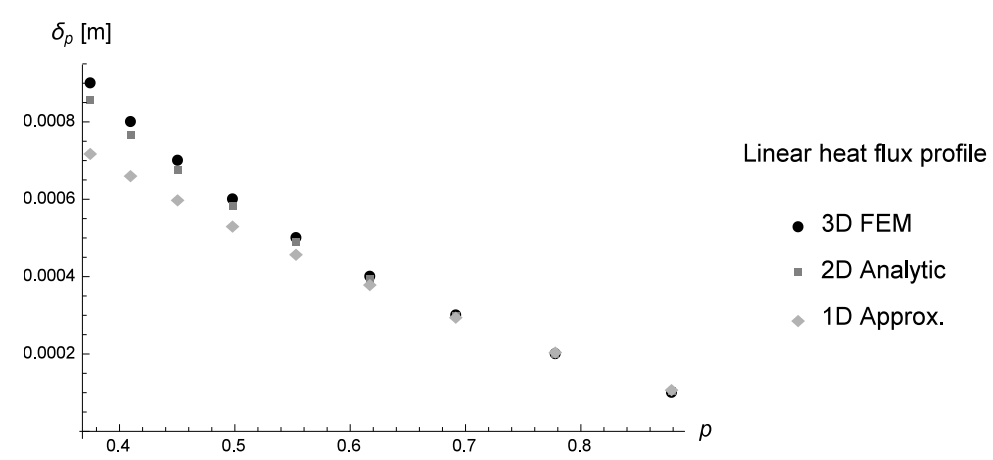

Fig. 10 Depth of thermal penetration given by [30] compared to $1 \mathrm{D}$ and $2 \mathrm{D}$ analytical approaches.

In Fig. 10, the two-dimensional approach departs from the three-dimensional FEM data because, in the latter, the finite width of the grinding wheel (which 
is narrower than the workpiece width) makes the temperature field not exactly uniform along the $z$-axis, being a little bit greater at the center of the wheel than at both the sides of it. Therefore, in the three-dimensional simulation, the finite width of the heat source (i.e. the grinding wheel width) makes the maximum temperature at a given depth slightly higher than in the twodimensional model, in which the temperature field is supposed to be uniform along the $z$-axis. Equivalently, the depth of thermal penetration $\delta_{p}$ is slightly greater in the three-dimensional approach for a given temperature (or for a given $p$ ) beneath the surface, as it is seen in Fig. 10.

\section{Conclusions}

The definition of the depth of thermal penetration in one-dimensional heat transfer problems has been applied to the two-dimensional heat transfer models in straight grinding, both in the dry as in the wet case. In wet grinding, the one-dimensional boundary-value problem has been solved combining a boundary condition of the second kind with other of the third kind. The latter seems not to be reported in the most common literature. For the two-dimensional boundary-value problem, a set of two non-linear equations has been derived in order to solve the depth of thermal penetration. These equations depend on the heat flux profile considered (i.e. constant, linear, triangular and parabolic, as reported in the literature), as well as whether we apply or not coolant onto the workpiece surface. Since these Eqs. have to be solved numerically, an initial seed has been derived in Eqs. (63) and (68), as well as a procedure to plot the graphs of Figs. 4-9.

In dry grinding, the one-dimensional approximation of the depth of thermal penetration provides accurate results when we consider a moderate percentage in the temperature fall beneath the surface, i.e. $0.4 \leq p \leq 1$. From a physical point of view, this is to be expected, since nearby the heat source, the heat transfer is mostly conducted perpendicularly to the surface. The latter is true in wet grinding, if we consider a constant heat flux profile and a high Peclet number, i.e. $\mathrm{Pe}>5$. This is consistent with the derivation given in Section 3 .

Also, we have compared the one- and two-dimensional approaches of the depth of thermal penetration with a temperature field in dry grinding simulated by a three-dimensional FEM analysis (see Ref. [30]), obtaining very good results for both approaches when $0.6 \leq p \leq 1$. It is worth noting that the twodimensional approach exhibits a better performance than the one-dimensional one.

Finally, we can conclude that the one-dimensional approximation is useful when we consider a moderate temperature fall beneath the surface, because this one is quite accurate and easy to compute, unlike to the calculation of depth of thermal penetration in the two-dimensional approach. As a direct application of the latter, we highlight that this analytical approach can estimate very easily the thickness of the heat treated zone in the grind-hardening 
process, as well as to set the domain of the temperature field, both in FEM simulations as in experimental measurements, during the grinding process.

Acknowledgements The authors wish to thank the financial support received from Universidad Católica de Valencia under grant PRUCV/2015/612.

\section{A One-dimensional solution for wet grinding}

We have to solve the following boundary-value problem:

$$
\begin{aligned}
\frac{\partial T(t, y)}{\partial t} & =k \frac{\partial^{2} T(t, y)}{\partial y^{2}}, \\
T(0, y) & =T_{\infty}, \quad y>0, \\
\frac{\partial T}{\partial x}(t, 0) & =-q_{0}+h_{0}\left[T(t, 0)-T_{\infty}\right], \quad t>0 .
\end{aligned}
$$

Performing the change of variables,

$$
\vartheta(t, y)=T(t, y)-T_{\infty}+q_{0} y,
$$

the problem stated in Eqs. (87)-(89) become

$$
\begin{aligned}
\frac{\partial \vartheta}{\partial t} & =k \frac{\partial^{2} \vartheta}{\partial y^{2}}, \\
\frac{\partial \vartheta}{\partial y}(t, 0) & =h_{0} \vartheta(t, 0), \\
\vartheta(0, y) & =q_{0} y .
\end{aligned}
$$

In Ref. [9, Eqn. 14.2(7)], the solution to the problem

$$
\begin{aligned}
\frac{\partial v}{\partial t} & =k \frac{\partial^{2} v}{\partial x^{2}}, \\
\frac{\partial v}{\partial y}(t, 0)-h v(t, 0) & =h \phi(t), \\
v(0, x) & =f(x),
\end{aligned}
$$

is given by

$$
\begin{aligned}
& v(t, x) \\
= & \int_{0}^{\infty} f(\xi)\left\{\frac{e^{-(x-\xi)^{2} / 4 k t}+e^{-(x+\xi)^{2} /(4 k t)}}{2 \sqrt{\pi k t}}\right. \\
& \left.-h e^{k t h^{2}+h(x+\xi)} \operatorname{erfc}\left(\frac{x+\xi}{2 \sqrt{k t}}+h \sqrt{k t}\right)\right\} d \xi \\
& +k h \int_{0}^{t} \phi(\tau)\left\{\frac{e^{-x^{2} / 4 k(t-\tau)}}{\sqrt{\pi k(t-\tau)}}\right. \\
& \left.-h e^{k h^{2}(t-\tau)+h x} \operatorname{erfc}\left(\frac{x}{2 \sqrt{k(t-\tau)}}+h \sqrt{k(t-\tau)}\right)\right\} d \tau .
\end{aligned}
$$


Therefore, applying the solution given in Eq. (94) to the boundary-value problem stated in Eqs. (91)-(93), taking $\phi(t)=0$ and $f(y)=q_{0} y$, we have

$$
\begin{aligned}
& \vartheta(t, y) \\
= & q_{0}\left\{\frac{1}{2 \sqrt{\pi k t}} \int_{0}^{\infty}\left(e^{-(y-\xi)^{2} / 4 k t}+e^{-(y+\xi)^{2} / 4 k t}\right) \xi d \xi\right. \\
& \left.-h_{0} e^{k t h_{0}^{2}} \int_{0}^{\infty} e^{h_{0}(y+\xi)} \operatorname{erfc}\left(\frac{y+\xi}{2 \sqrt{k t}}+h_{0} \sqrt{k t}\right) \xi d \xi\right\} .
\end{aligned}
$$

The integral given in Eq. (96), denoted by $I_{1}$ hereafter, can be calculated by using the following result (see Ref. [31, Eqn. 3.462.5]),

$$
\begin{aligned}
& \int_{0}^{\infty} x e^{-\mu x^{2}-2 \nu x} d x=\frac{1}{2 \mu}-\frac{\nu}{2 \mu} e^{\nu^{2} / \mu} \operatorname{erfc}\left(\frac{\nu}{\sqrt{\mu}}\right), \\
& |\arg \nu|<\frac{\pi}{2}, \operatorname{Re} \mu>0,
\end{aligned}
$$

thereby

$$
\begin{aligned}
I_{1} & =\frac{1}{2 \sqrt{\pi k t}} \int_{0}^{\infty}\left(e^{-(y-\xi)^{2} / 4 k t}+e^{-(y+\xi)^{2} / 4 k t}\right) \xi d \xi \\
& =2 \sqrt{\frac{k t}{\pi}} e^{-y^{2} / 4 k t}+y \operatorname{erfc}\left(\frac{y}{2 \sqrt{k t}}\right)
\end{aligned}
$$

The infinite integral given in Eq. (97), denoted by $I_{2}$, can be expressed in terms of the indefinite integral $I_{2}(\xi)$ as

$$
I_{2}=\lim _{\xi \rightarrow \infty} I_{2}(\xi)-I_{2}(0),
$$

In order to calculate $I_{2}(\xi)$, perform the substitution $u=\frac{y+\xi}{2 \sqrt{k t}}+h_{0} \sqrt{k t}$ and set $\alpha=$ $2 h_{0} \sqrt{k t}$, to arrive at

$$
\begin{aligned}
I_{2}(\xi)=- & h_{0} e^{k t h_{0}^{2}} \int e^{h_{0}(y+\xi)} \operatorname{erfc}\left(\frac{y+\xi}{2 \sqrt{k t}}+h_{0} \sqrt{k t}\right) \xi d \xi \\
=- & \alpha \frac{\alpha}{h_{0}} e^{-\alpha^{2} / 4} \int u e^{\alpha u} \operatorname{erfc}(u) d u \\
& \left.-(\alpha \sqrt{k t}+y) \int e^{\alpha u} \operatorname{erfc}(u) d u\right\}
\end{aligned}
$$

Both integrals given in Eqs. (100) can be calculated with the following results (see Ref. [32, Eqns. 1.5.2(3)-(4)]):

$$
\int e^{b y} \operatorname{erfc}(a y) d y=\frac{e^{b y}}{b} \operatorname{erfc}(a y)+\frac{e^{b^{2} / 4 a^{2}}}{b} \operatorname{erfc}\left(a y-\frac{b}{2 a}\right)
$$

and

$$
\begin{aligned}
& \int y e^{b y} \operatorname{erfc}(a y) d y \\
= & \frac{e^{b y}}{b}\left(y-\frac{1}{b}\right) \operatorname{erfc}(a y) \\
& +\frac{e^{b^{2} / 4 a^{2}}}{b}\left[\left(\frac{1}{2 a^{2}}-\frac{1}{b}\right) \operatorname{erfc}\left(a y-\frac{b}{2 a}\right)-\frac{e^{-(2 a y-b / a)^{2} / 4}}{a \sqrt{\pi}}\right] .
\end{aligned}
$$


Therefore, Eq. (100) is calculated as

$$
\begin{aligned}
I_{2}(\xi)= & 2 \sqrt{\frac{k t}{\pi}} e^{-(y+\xi)^{2} / 4 k t}+\frac{1+h_{0} y}{h_{0}} \operatorname{erf}\left(\frac{y+\xi}{2 \sqrt{k t}}\right) \\
& +\frac{1-h_{0} y}{h_{0}} e^{h_{0}^{2} k t+h_{0}(y+\xi)} \operatorname{erfc}\left(\frac{y+\xi}{2 \sqrt{k t}}+h_{0} \sqrt{k t}\right) .
\end{aligned}
$$

Notice that since $y, t, k, h_{0}>0$, we have

$$
\begin{aligned}
\lim _{\xi \rightarrow \infty} I_{2}(\xi) & =\frac{1+h_{0} y}{h_{0}}-\lim _{\xi \rightarrow \infty} \xi e^{h_{0} \xi} \operatorname{erfc}\left(\frac{\xi}{2 \sqrt{k t}}\right) \\
& =\frac{1+h_{0} y}{h_{0}},
\end{aligned}
$$

where we have used the asymptotic expansion (see Ref. [27, Eqn. 7.12.1]),

$$
\operatorname{erfc}(z) \sim \frac{e^{-z^{2}}}{\sqrt{\pi} z} \sum_{m=0}^{\infty}(-1)^{m} \frac{(2 m-1) ! !}{\left(2 z^{2}\right)^{m}}, \quad z \rightarrow \infty .
$$

Therefore, taking into account Eqs. (101) and (102) in Eq. (99), we have

$$
\begin{aligned}
I_{2}= & \frac{1+h_{0} y}{h_{0}} \operatorname{erfc}\left(\frac{y}{2 \sqrt{k t}}\right)-2 \sqrt{\frac{k t}{\pi}} e^{-y^{2} / 4 k t} \\
& -\frac{e^{h_{0}^{2} k t+h_{0} y}}{h_{0}} \operatorname{erfc}\left(\frac{y}{2 \sqrt{k t}}+h_{0} \sqrt{k t}\right) .
\end{aligned}
$$

Substituting back in Eqs. (95) and (90) the results obtained in Eqs. (98) and (103), we finally arrive at

$$
\begin{aligned}
& T(t, y)-T_{\infty} \\
= & \frac{q_{0}}{h_{0}}\left\{\operatorname{erfc}\left(\frac{y}{2 \sqrt{k t}}\right)-e^{h_{0}^{2} k t+h_{0} y} \operatorname{erfc}\left(\frac{y}{2 \sqrt{k t}}+h_{0} \sqrt{k t}\right)\right\} .
\end{aligned}
$$

\section{References}

1. Malkin S, Guo C (2008) Grinding Technology: Theory and Application of Machining with Abrasives. Industrial Press Inc, New York

2. Jaeger JC (1942) Moving Sources of Heat and the Temperature at Sliding Contracts. P Roy Soc NS Wales 76:204-224

3. Li J, Li JCM (2005) Temperature distribution in workpiece during scratching and grinding. Mat Sci Eng A-Struct 409(1):108-119. doi: j.msea.2005.07.057

4. Zarudi I, Zhang LC (2002) A revisit to some wheel-workpiece interaction problems in surface grinding. Int J Mach Tool Manu 42:905-913. doi: 10.1016/S0890-6955(02)00024-

5. Yan XT (2002) On the Penetration Depth in Fourier Heat Conduction. 8th AIAA/ASME Joint Termophysics and Heat Transfer Conference, Saint Louis, MO

6. Takazawa K (1966) Effects of grinding variables on surface structure of hardened steel. Bull Japan Soc Prec Eng 2(1):14-21

7. Brinksmeier E, Brockhoff T (1996) Utilisation of grinding heat as a new heat treatment process. CIRP Ann Manuf Tech 45 (1):283-286. doi: 10.1016/S0007-8506(07)63064-9

8. Salonitis K, Chryssolouris G (2007) Cooling in grind-hardening operations. Int J Adv Manuf Technol 33(3-4):285-297. doi: 10.1007/s00170-006-0467-9

9. Carslaw HS, Jaeger JC (1959) Conduction of heat in solids, 2nd ed. Clarendon Press, Oxford 
10. Skuratov D, Ratis Y, Selezneva I, Pérez J, Fernández de Córdoba P, Urchueguía J (2007) Mathematical modelling and analytical solution for workpiece temperature in grinding. Appl Math Model 31:1039-1047. doi: 10.1016/j.apm.2006.03.023

11. Lavine AS, Malkin S, Jen TC (1989) Thermal aspects of grinding with CBN wheels. CIRP Annals-Manu Tech 38:557-560. doi: 10.1016/S0007-8506(07)62768-1

12. Guo C, Malkin S (1995) Analysis of energy partitions in grinding. J Eng Ind-T ASME 117:55-61. doi: 10.1115/1.2803278

13. Sauer WJ (1971) Thermal aspects of grinding. Dissertation, Carnegie-Mellon University

14. Guo C, Wu Y, Varghese V, Malkin S (1999) Temperatures and energy partition for grinding with vitrified CBN wheels. CIRP Annals-Manu Tech 42:247-250 doi: 10.1016/S0007-8506(07)63176-X

15. Mahdi M, Zhang L (1998) Applied mechanics in grinding-VI. Residual stresses and surface hardening by coupled thermo-plasticity and phase transformation. Int J Mach Tool Manu 38:1289-1304. doi: 10.1016/S0890-6955(97)00134-X

16. Rowe WB, Black SC, Mills B, Qi HC, Morgan MN (1995) Experimental investigation of heat transfer in grinding. CIRP Annals-Manu Tech. doi: 10.1016/S0007-8506(07)623361

17. Knothe K, Liebelt S (1995) Determination of temperatures for sliding contact with applicatios for wheel-rail systems. Wear 189:91-99. doi: 10.1016/0043-1648(95)06666-7

18. Beck JV, Cole KD, Haji-Sheikh A, Litkouhi B (2010) Heat conduction using Green's functions, 2nd ed. CRC Press, Boca Raton

19. González-Santander JL, Martín G (2015) A theorem for finding maximum temperature in wet grinding. Math Probl Eng 2015. doi: 10.1155/2015/150493

20. González-Santander JL, Valdés Placeres JM, Isidro JM (2011) Exact solution for the time-dependent temperature field in dry grinding: application to segmental wheels. Math Probl Eng 2011. doi: 10.1155/2011/927876

21. González-Santander JL (2016) Maximum Temperature and Relaxation Time in Wet Surface Grinding for a General Heat Flux Profile. Math Probl Eng 2016. doi: $10.1155 / 2016 / 5387612$

22. González-Santander JL (2014) Calculation of an integral arising in dry flat grinding for a general heat flux profile. Application to maximum temperature evaluation. J Eng Math 88:137-160. doi: 10.1007/s10665-013-9684-z

23. DesRuisseaux NR, Zerkle RD (1970) Temperature in Semi-lnfinite and Cylindrical Bodies Subjected to Moving Heat Sources and Surface Cooling. J Heat Trans-T ASME 92(3):456-464. doi: 10.1115/1.3449689

24. Takahasi H, Mori M (1973) Double exponential formulas for numerical integration. Pub Res I Math Sci 9:721-741. doi: 10.2977/prims/1195192451

25. González-Santander JL, Martín González G (2015) Closed form expression for the surface temperature in wet grinding. Application to maximum temperature evaluation. J Eng Math 90(1):173-193. doi: 10.1007/s10665-014-9716-3

26. Oldham KB, Myland J, Spanier J (2010) An atlas of functions: with equator, the atlas function calculator. Springer Science \& Business Media, New York. doi: 10.1007/978-0387-48807-3

27. Olver FWJ, Lozier DW, Boisvert RF, Clark CW (2010) NIST Handbook of Mathematical Functions. Cambridge University Press, New York

28. Murav'ev VI, Yakimov AV, Chernysev AV (2003) Effect of deformation, welding, and electrocontact heating on the properties of titanium alloy VT20 in pressed and welded structures. Met Sci Heat Treat 45:419-422. doi: 10.1023/B:MSAT.0000019194.64623.48

29. Lavine AS (1988) A Simple Model for Convective Cooling During the Grinding Process. J Eng Ind-T ASME 110:1-6. doi: 10.1115/1.3187837

30. Wang X, Yu T, Sun X, Shi Y, Wang W (2016) Study of 3D grinding temperature field based on finite difference method: considering machining parameters and energy partition. Int J Adv Manuf Tech 84(5-8):915-927. doi: 10.1007/s00170-015-7757-z

31. Gradsthteyn IS, Ryzhik IM (2007) Table of integrals, series and products. Seventh edition. Academic Press Inc, New York

32. Prudnikov AP, Brychkov YA, Marichev OI (1986) Integrals and Series. Vol 2: Special Functions. Gordon and Breach, New York 